\title{
Retos de los docentes universitarios para el diseño de experiencias virtuales educativas en pandemia
}

\section{Challenges for university teachers in the design of virtual educational experiences during the pandemic}

\author{
Ricardo Navarro ${ }^{1}$ \\ Pontificia Universidad Católica del Perú \\ ricardo.navarro@pucp.pe \\ ORCID: https://orcid.org/0000-0002-7069-9780 \\ Renato López² \\ Pontificia Universidad Católica del Perú \\ a20170968@pucp.edu.pe \\ ORCID: https://orcid.org/0000-0002-6749-2025

\section{Gloria Caycho ${ }^{3}$} \\ Pontificia Universidad Católica del Perú \\ gloria.caychod@pucp.edu.pe \\ ORCID: https://orcid.org/0000-0002-3138-783X
}

Citar como: Navarro, R., López, R. y Caycho, G. (2021). Retos de los docentes universitarios para el diseño de experiencias virtuales educativas en pandemia. Desde el Sur, 13(2), e0017.

\section{RESUMEN}

Como respuesta a la emergencia sanitaria, las instituciones de educación superior han tenido que migrar forzosamente a la virtualidad. Esto ha impactado en los actores educativos y los procesos de enseñanza-aprendizaje. En este artículo se analizarán las características particulares de la educación virtual y los principios de la enseñanza en este contexto. Asimismo, se describirán propuestas para diseñar experiencias educativas virtuales considerando las teorías motivacionales del aprendizaje, y el diseño instruccional aplicado a la educación virtual. En esta misma línea, se discuten los retos que impone la pandemia para la

1 Licenciado en Psicología Educacional con maestría en Cognición, Aprendizaje y Desarrollo por la Pontificia Universidad Católica del Perú (PUCP).

2 Estudiante de Psicología en la PUCP, coordinador académico del MEPPCi, agrupación estudiantil de estudiantes de Psicología.

3 Estudiante de Psicología en la PUCP. Pasante del área de investigación del grupo Avatar PUCP. 
educación universitaria y se proponen líneas de acción para la creación de experiencias educativas no presenciales. Se concluye que existe una necesidad imperante de capacitar a los profesores de tal manera que puedan usar las distintas herramientas digitales a su disposición y reconocer la unicidad de la educación virtual en contraposición a las clases tradicionales.

\section{PALABRAS CLAVE}

Educación a distancia, tecnología de la información, enseñanza superior, docente

\section{ABSTRACT}

In response to the health emergency, higher education institutions had to forcibly migrate to virtual education. This has impacted the educational context, both in terms of teaching and learning. The following article analyzes the particular features of virtual education and the principles of teaching in this context. Also, proposals are described for the designing of virtual educational experiences that take into account motivational theories, learning theories, and instructional design applied to e-learning. Also, the challenges for university education posed by the pandemic are discussed, and lines of action are proposed for the creation of e-learning experiences. It is concluded that there is an urgent need for the training of teachers in the use of the different available tools for virtual education, and for recognition of the unique character of virtual education, compared to traditional in-person learning.

\section{KEYWORDS}

distance education, information technology, higher education, teachers

\section{Migración a la virtualidad por la pandemia}

En un contexto político y social incierto, el Perú se sitúa como uno de los países de Latinoamérica más afectados por la pandemia debido a la covid-19. Si bien es cierto que antes de la aparición de este virus el Perú era un país relativamente estable en términos económicos, su sistema de salud siempre se ha caracterizado por ser uno de los más endebles del mundo (Figallo et al., 2020). Esto, sumado a las profundas desigualdades económicas y sociales, ha generado una serie de crisis en las diferentes instituciones públicas y privadas del país (Varas et al., 2020). Asimismo, 
el aislamiento social obligatorio y preventivo ha supuesto otra serie de retos y dificultades en términos sociales y emocionales para los diferentes sectores de la población. En ese sentido, el sector educativo no ha sido ajeno a estos cambios, puesto que se ha visto severamente afectado por el distanciamiento social y las medidas de salud para evitar el contagio. Debido a ello, gran parte de los entornos educativos tuvieron que migrar a la virtualidad, lo que trajo consigo algunas complicaciones en la enseñanza brindada por parte de las instituciones educativas. Se podría realizar un análisis extenso sobre las consecuencias en la enseñanza que sufrió la Educación Básica Regular (EBR); sin embargo, el objetivo del presente ensayo es analizar las características de la educación virtual, para así plantear algunas pautas para el diseño de experiencias educativas virtuales y discutir los retos que impone la pandemia para la educación universitaria.

Las universidades tuvieron que enfrentar una migración fortuita y no planificada de la enseñanza presencial a la virtual (Lovón y Cisneros, 2020), lo que implicó el rediseño de los contenidos de enseñanza presencial a una modalidad virtual, pese a que muchos de estos materiales existentes no habían sido creados para ser utilizados en un contexto virtual (García et al., 2020). Esta realidad genera matices de complejidad si se toma en cuenta que existen materias cuyas competencias deben ser desarrolladas mediante la experimentación práctica del laboratorio, el cual no puede ser emulado por la virtualidad (Perales, 2019). Es ante esta serie de desafíos que la educación superior privada y pública se adapta sobre la marcha a las exigencias de los entornos virtuales de aprendizaje, a través de la reprogramación de sus calendarios académicos y la digitalización de sus contenidos, con el fin de implementar la educación en línea (Cepal-Unesco, 2020; Figallo et al., 2020). Así, el personal docente de las universidades se enfrentó a un reto sin precedentes: rediseñar todas sus experiencias educativas presenciales a un contexto virtual.

No es posible comparar una experiencia educativa presencial a una virtual, y querer replicar escenarios presenciales (como dictar clases) en medios virtuales es inadecuado o, en todo caso, limitado. En ese sentido, las diferencias entre el entorno virtual y el presencial radican en el tipo de actividades realizadas, los medios y las formas de organización del aprendizaje (Vialart, 2020). Por ello, es importante enfocarse en la manera más adecuada de diseñar contenido digital que asegure y promueva el aprendizaje del alumnado. En otras palabras, este contexto impulsa al cuerpo docente a construir nuevas prácticas educativas que potencien el aprendizaje del alumnado a través del uso creativo de las TIC (Arias y Merino, 2016). 


\section{Prácticas educativas e innovación}

Parte sustancial del proceso de enseñanza-aprendizaje, tanto en entornos virtuales como presenciales, son las prácticas educativas que utilizan los docentes. Se puede entender a las prácticas educativas como el conjunto de actividades que se realizan dentro de un contexto de enseñanza para influir en el proceso de aprendizaje de los estudiantes (Canedo et al., 2018; Chávez y Jaramillo, 2014; Coll et al., 2008). Estas prácticas deben tener en cuenta las características de los estudiantes, sus interacciones con otros (compañeros y docentes) o con un objeto de aprendizaje, el rol que cumplen los estudiantes dentro de una actividad específica, así como el tiempo asignado para la realización de dicha actividad (Chávez y Jaramillo, 2014). Una característica que vale la pena resaltar es que toma en cuenta las interacciones del estudiante como parte primordial de su aprendizaje. Esta concepción posee una notoria influencia constructivista, ya que la interacción con el medio que rodea al estudiante (estudiantedocente, estudiante-estudiante, estudiante-objeto de aprendizaje) es esencial (Coloma y Tafur, 1999). Bajo este enfoque, el aprendizaje no significa internalizar una copia de la realidad, tal como es entendido desde un paradigma asociacionista; sino se entiende como un proceso activo en el que cada individuo interpreta la información externa usando sus esquemas de conocimiento previos, que se van complejizando conforme interactúan con su entorno (Coloma y Tafur, 1999).

Por otro lado, las prácticas educativas innovadoras - o la innovación educativa en general- implica el uso de nuevas herramientas o dinámicas para la enseñanza (Serdyukov, 2017). Sin embargo, hay que recalcar que dicha innovación no necesariamente trae consigo conceptos educativos nuevos, sino que se basan en las teorías de aprendizaje existentes para potenciar las intervenciones educativas. En muchos casos estas innovaciones buscan incorporar nuevas tecnologías para potenciar las metodologías de enseñanza del docente (Serdyukov, 2017). Asimismo, para usar adecuadamente una práctica educativa innovadora se debe tener en cuenta la gestión de los contenidos enseñados, las evaluaciones que se realizan, las metodologías de enseñanza adecuadas según el contenido, los recursos (herramientas educativas) y promover la participación de los estudiantes (Canedo et al., 2018). En ese sentido, Vargas et al. (2016) recomiendan tomar en cuenta los conocimientos previos de los estudiantes, crear retos de aprendizaje, contextualizar los contenidos, promover la autonomía y autorregulación del aprendizaje de los estudiantes, y fomentar el trabajo cooperativo. Además, se reconocen prácticas educativas que incorporan hardware y software destinados a mejorar los aprendizajes e incrementar la motivación de los estudiantes (por ejemplo, los estudios de 
Buabeng et al., 2018; Sadik, 2017; Xu y Du, 2019). Dentro de las diversas experiencias educativas de este tipo, se puede destacar la enseñanza virtual.

Si bien esta innovación educativa aborda las mismas características mencionadas anteriormente, se complejiza porque requiere una aproximación distinta a la de una enseñanza presencial. Así, es importante entender lo siguiente: en un contexto de educación virtual, no se está utilizando una herramienta innovadora para enseñar en el salón de clase; sino que toda la experiencia educativa ha cambiado, lo que representa un reto para los docentes y estudiantes, así como un cambio de paradigma en la enseñanza.

\section{¿Qué es la educación virtual?}

La educación virtual es un área de investigación muy estudiada en los últimos años y, a raíz de la pandemia, se ha vuelto absolutamente necesaria abordarla. Tal es así que el campo de discusión académica alrededor de este concepto abarca diferentes áreas temáticas, desde el diseño de experiencia del usuario (user experience), el diseño instruccional de las sesiones de los cursos virtuales basados en los conceptos que se enseñan, las diferentes estrategias aplicadas según la temática abordada (psicopedagogía), así como sus beneficios y limitaciones en el aprendizaje del estudiante. Por ello, se hace necesario delimitar adecuadamente qué se quiere analizar y proponer en estos campos, lo que requiere un trabajo interdisciplinario que va más allá del simple manejo de contenidos teóricos.

En primer lugar, sería prudente definir qué es la educación virtual. Para varios autores, la educación virtual es un tipo de educación a distancia que utiliza tecnologías como medio para establecer los espacios de aprendizaje (Arkorful y Abaidoo, 2015; Rodrigues et al., 2019; Rosenberg, 2001). La literatura sobre el tema ha delimitado la enseñanza virtual en cuestiones ligadas al hardware y software más que al contenido en sí (por ejemplo, m-learning y e-learning) (Rodrigues et al., 2019); sin embargo, estas delimitaciones son utilizadas para describir características específicas de aplicaciones prácticas en la enseñanza, mas no difieren de los conceptos y fenómenos transversales ligados al aprendizaje que ocurren en los estudiantes. Por tal motivo, y porque cada apartado requeriría un análisis más extenso del que este texto pretende abordar, el presente ensayo se enfocará en las características generales de la educación virtual y el diseño de experiencias educativas en general.

Debido a que se ha reconocido a las prácticas educativas como un aspecto importante de la enseñanza, se hace relevante abordar el diseño de las sesiones virtuales y las estrategias que se pueden y deben usar en 
un curso virtual. Aunque existen preceptos transversales en la enseñanza presencial y virtual que deben manejarse, estos no se presentan de la misma manera en ambos. Un curso virtual posee otras características y herramientas que deben ser tomadas en cuenta, y es responsabilidad del docente saber cómo aprovecharlas.

Por ello, es necesario tener claridad sobre la dinámica de la educación virtual, que difiere en mayor medida de una clase presencial tradicional. Así, un aspecto resaltante de la educación virtual es el distanciamiento físico entre el estudiante y el docente, que recurren a espacios virtuales para generar procesos de enseñanza-aprendizaje (Rodrigues et al., 2019). En consecuencia, se hace necesario el uso de software y hardware para participar en estos espacios virtuales, lo que permitirá intercambiar información, recursos de aprendizaje, dinamizar los procesos de comunicación y generar interacción entre el estudiante y los contenidos de aprendizaje (Rodrigues et al., 2019). Sin embargo, esta interacción no es la misma que en espacios presenciales, pues dichas interacciones no son viables. Otro aspecto importante de recalcar de la educación virtual son los momentos donde ocurren los procesos de enseñanza-aprendizaje, los cuales pueden ser sincrónicos o asincrónicos. El primero hace referencia a espacios donde el estudiante participa en actividades de aprendizaje en un tiempo determinado con el profesor, quien realiza las actividades en dicho momento. El segundo hace referencia a procesos de aprendizaje que ocurren en cualquier otro momento, con material o actividades que el docente deja para el estudiante, y este puede revisarlo cuando crea conveniente. Esta diferenciación genera que ocurran ciertos procesos psicológicos distintos a los que normalmente se encontraría en un contexto presencial.

\section{Procesos psicológicos y el diseño de experiencias educativas virtuales}

Durante la enseñanza virtual, hay procesos psicológicos que deben ser tomados en cuenta sobre otros y que, tal vez, tenían otro tipo de actividades para fomentarlos. Un ejemplo de ello es la motivación del estudiante en entornos virtuales, que se puede fomentar de distinta manera que en la presencialidad.

La motivación es uno de los factores más importantes que afectan la conducta, y puede tener un impacto en cómo y por qué aprendemos (Deci y Ryan, 2000; Firat et al., 2018). Diversos estudios mencionan la importancia de la motivación intrínseca para el aprendizaje (Deci y Ryan, 2000; Eom, 2019), lo cual hace que fomentar este tipo de motivación sea parte importante de cualquier proceso de enseñanza. De igual forma, 
existe evidencia de que los niveles de motivación intrínseca de los estudiantes por aprender en cursos virtuales son altos (Firat et al., 2018). Sin embargo, esto ocurre no porque el estudiante esté aprendiendo de manera virtual, sino que existen algunos requisitos en el diseño de la experiencia que son cumplidos. Uno de estos requisitos importantes a recalcar es el de la autonomía en el proceso de aprendizaje.

La autonomía hace referencia al control que una persona tiene sobre sus propias decisiones (Deci y Ryan, 2000). Existe todo un campo de estudio extenso sobre los beneficios de fomentar una conducta motivada autónomamente en los estudiantes; y su importancia en el aprendizaje virtual, así como su efecto en el aprendizaje de los estudiantes (Eom, 2019; Ratnaningsih et al., 2020; Rahm et al., 2021). Si el estudiante no está motivado intrínsecamente por aprender los contenidos de un curso virtual, es más propenso a no abordar adecuadamente los objetos de aprendizaje, ni prestar atención a los contenidos del curso (Eom, 2019; Rahm et al., 2021). Para motivar a los estudiantes de cursos virtuales se debe mantener cierto nivel de interés en ellos, mediante el fomento de la autonomía, y promover la interacción entre el estudiante y los objetos de aprendizaje. Así, cursos virtuales que fomentan la interacción y autonomía en los estudiantes generan que los estudiantes se sientan más motivados por aprender los temas de enseñanza (Eom, 2019).

Asimismo, existen algunos factores sociales que pueden regular las conductas de los estudiantes cuando afrontan una tarea en grupo. En estudios que abordan el proceso de enseñanza-aprendizaje, se toma en cuenta que los trabajos grupales pueden ser una mejor manera de fomentar el aprendizaje en contextos virtuales (Gillies, 2003; Retnowati et al., 2017). Asimismo, estudios sobre el tema (Barari et al., RezaeiZadeh, Khorasani y Alami, 2020; Retnowati et al., 2017) refieren que este tipo de aproximaciones pedagógicas posee mejores resultados académicos que intervenciones donde el estudiante debe aprender de manera estática, sin interacciones. En ese sentido, los estudios argumentan que estos beneficios se pueden explicar a partir de la teoría socioconstructivista, que enfatiza el aprendizaje facilitado a partir de la socialización y la colaboración en actividades académicas, en las que el conocimiento se construye a partir de la interacción con otras personas (Schreiber y Valle, 2013; Retnowati et al., 2017; Kumi, 2018; Barari et al., 2020). Es decir, no es suficiente proponer actividades de aprendizaje que exijan al estudiante solo leer textos o ver videos sobre un tema. Tiene que haber un espacio que fomente la autonomía del estudiante en su propio aprendizaje y se pueda construir el conocimiento. 
Por otro lado, es importante abordar el diseño de las actividades de la enseñanza virtual. Para ello, es necesario hacer una revisión conceptual del diseño instruccional y cómo puede ser aplicado en una experiencia virtual.

El diseño instruccional es un proceso pedagógico que estructura actividades para crear una experiencia de aprendizaje, con el objetivo de hacer más amigable, eficaz y eficiente el proceso educativo (Tarazona, 2012). Asimismo, en un contexto virtual, el diseño instruccional comprende las fases de análisis, diseño, desarrollo, implementación y evaluación de las experiencias de aprendizaje (Tarazona, 2012; Chen, 2016). Luego, se decide la organización de los contenidos para el diseño de la clase virtual, así como las tecnologías adecuadas para realizar las actividades diseñadas (Chen, 2016). De este modo, el uso del diseño instruccional para cursos virtuales tiene como objetivo que la enseñanza se adapte a las necesidades del grupo de estudiantes en la virtualidad, por lo cual tiende a alejarse de las experiencias de aprendizaje tradicionales que se ofrecen en una modalidad presencial. Según Martínez (2008), existen diferencias sustanciales entre ambas, más allá de la presencia física de los estudiantes y docentes. Por ejemplo, dentro de un ambiente físico, el estudiante puede prestar atención a las indicaciones del docente y realizar preguntas de manera más natural que en la virtualidad. Además, son comunes el uso de notas y la interacción social posterior a la clase, donde los estudiantes pueden realizar comentarios o compartir dudas sobre lo visto en el aula. La virtualidad, por otro lado, permite que el estudiante pueda controlar el orden en que accede a los contenidos de aprendizaje y repetir los contenidos para su posterior análisis. Sin embargo, existen limitaciones importantes que afectan el proceso de enseñanza, como la comunicación con los docentes y otros estudiantes.

Por otro lado, un ambiente virtual de aprendizaje se caracteriza por usar algunos sistemas de manejo del aprendizaje como Canvas, Paideia o Blackboard. Estas plataformas no solo facilitan la gestión de los contenidos, sino también recolectan datos que permiten analizar el compromiso y rendimiento de los estudiantes, y mejoran la enseñanza según la retroalimentación (Alves et al., 2017). Estas plataformas son la base de la infraestructura de la enseñanza virtual, y sin ellas sería complicado realizar una experiencia educativa adecuada.

Por otro lado, el intercambio de conocimientos típicamente es asincrónico y ello permite que el proceso sea flexible. Es el estudiante quien decide sus horarios y su ritmo de aprendizaje (Tran, 2016). Respecto a la comunicación entre profesores y estudiantes, puede darse a través del correo, del foro de preguntas y de las sesiones de video (Martínez, 2008). 
Por esta razón y por la naturaleza de la modalidad virtual, es indispensable que el estudiante tenga conexión de Internet y un dispositivo para acceder a la plataforma.

En esa misma línea, es importante atender la problemática del desconocimiento por parte del profesorado de recursos educativos en la virtualidad (Bracho y Bracho, 2020). No obstante, existen diversos recursos como los videos, las animaciones, los cuestionarios, los juegos, entre otros, que podrían enriquecer el ecosistema educativo virtual (Ferri et al., 2020). En adición, Heng y Sol (2020) proponen hacer dinámico, interesante e interactivo el proceso de aprendizaje, a través de foros en redes sociales y otras plataformas digitales, que puedan dar retroalimentación continua y oportuna al alumnado. Entonces, el diseño de las sesiones virtuales debe tener en cuenta el uso estratégico de las funcionalidades de estos softwares. Además, deben fomentar la interacción con otros estudiantes, lo que permite el intercambio de información relacionada con los contenidos temáticos estudiados. En ese sentido, plantear actividades que fomenten estos escenarios es deseable y necesario (Paechter y Maier, 2010). Se deben fomentar entornos educativos en los cuales el estudiante ponga en práctica lo que se ha enseñado, pues, de lo contrario, no tendrá mucho impacto en su aprendizaje (Pachter y Maier, 2010).

Es necesario señalar algunas características importantes al diseñar una clase virtual que no son replicables - o necesarias - en una presencial. Primero, se debe tener en cuenta que las actividades presenciales permiten un mayor número de interacciones, tanto académicas como sociales, dentro del salón de clase. Esto permite el uso de diversas actividades que facilitan la comprensión de un tema. Sin embargo, ello no ocurre en un contexto virtual, pues existen severas limitaciones en las interacciones de los estudiantes con el medio que los rodea. Para comenzar, el medio se limita a una pantalla, no a un espacio considerable como es el salón de clase. Esta limitación hace que el docente deba buscar otras maneras de generar interacción en los estudiantes.

Las tecnologías han permitido crear nuevas formas de enseñar, que han permitido el desarrollo de estrategias adecuadas a las necesidades de los estudiantes y han generado diversos tipos de formación virtual, ya sean cursos, diplomados y hasta posgrados (Rodrigues et al., 2019). A partir de lo explicado anteriormente, se pueden proponer algunas pautas que deben tenerse en cuenta en la educación virtual:

- Crear objetos de aprendizaje interactivos para explicar conceptos base de las clases que llevan los estudiantes, utilizando herramientas como Canvas, Genially, Socrative, etc. 
- Fomentar la interacción sincrónica entre el docente y los estudiantes, a fin de ahondar en los contenidos propuestos por los objetivos de aprendizaje.

- Fomentar actividades asincrónicas y sincrónicas entre los estudiantes.

- Generar espacios de aprendizaje que motiven a los estudiantes.

También deben considerarse otros aspectos importantes antes de diseñar una experiencia de enseñanza virtual. Así, se puede mencionar lo siguiente: organizar la estructura del curso de tal manera que facilite el aprendizaje de los estudiantes; delimitar adecuadamente los objetivos de aprendizajes; generar espacios de interacción entre el docente y los estudiantes, que permitan orientar los contenidos que el estudiante está aprendiendo, así como brindar retroalimentación; finalmente, fomentar la motivación (Rodrigues et al., 2019).

Esta revisión sobre los factores que se deben tener en cuenta en la educación virtual lleva a realizar un contraste con la realidad. ¿Cuáles son los retos que los docentes de las universidades deben afrontar en este cambio a la virtualidad?

\section{Retos que los docentes universitarios deben enfrentar en la enseñanza virtual}

Si bien la educación virtual en el contexto de emergencia sanitaria sigue representando un proceso de aprendizaje constante, tanto en estudiantes como en docentes, aún existen ciertos desafíos que se deben enfrentar. Por un lado, hay que tener en cuenta que existe una brecha digital y económica que se ha acentuado debido al contexto de crisis en pandemia. En relación con los estudiantes, solo en 2018 se reportó que únicamente el $57 \%$ de estudiantes peruanos contaba con acceso a Internet y el $53 \%$ con acceso a una computadora (Cepal-Unesco, 2020). Asimismo, alrededor del $80 \%$ de estudiantes que están en el cuartil socioeconómico más alto cuenta con un dispositivo que le permite acceder a Internet, mientras que solo entre el $10 \%$ y el $20 \%$ de los alumnos de los cuartiles más bajos cuenta con este tipo de dispositivos (Cepal-Unesco, 2020). Es esperable entonces que estas cifras se hayan intensificado como producto de la crisis actual, lo que muestra una deficiencia al acceso de tecnologías para recibir las clases virtuales (Marquina et al., 2020). Ante esta realidad, los docentes debieron tomar en consideración los recursos con los que cuentan sus estudiantes para adaptar sus prácticas educativas a estas limitaciones, lo que puede haber complicado más el tipo de diseño de sesiones virtuales utilizadas.

Cuando existe un diseño instruccional pauteado (que tiene en cuenta estas limitaciones), el entorno virtual es favorable para los aprendizajes y la 
satisfacción de los estudiantes con el servicio educativo (Niño et al., 2021). Es así como lo virtual no sería un problema por sí mismo, sino que este radicaría en las aproximaciones pedagógicas y metodológicas utilizadas en la educación virtual. En esa línea, se ha identificado que uno de los desafíos es que los profesores carecen de habilidades tecnológicas para desenvolverse adecuadamente en un entorno virtual (Ferri et al., 2020). Esta situación puede agravarse en los que tuvieron acceso a la tecnología recién a una edad avanzada (Parra et al., 2020), lo que convierte a la situación en un desafío particularmente difícil para ellos (Torres y García, 2019). Así, un problema que puede generarse en la implementación de cursos virtuales en educación superior es la falta de capacitación del personal en el uso de ciertas herramientas. Los resultados de esta carencia pueden ser perjudiciales para el aprendizaje del estudiante.

En la capacitación en metodologías virtuales es necesario reconocer la naturaleza distinta y el potencial de la modalidad virtual, así como la transformación de la experiencia educativa (Ferri et al., 2020). En este sentido, las instituciones educativas tendrían la responsabilidad de ofrecer soporte técnico y capacitaciones constantes a sus profesores, de modo que la autoeficacia en el uso de tecnologías para la enseñanza aumente e incida en el desenvolvimiento docente durante las clases. Además, vista la coyuntura actual, la modalidad virtual está lejos de ser un medio de transición, y es imperante que se construyan las bases necesarias que hagan llevadero este proceso.

De esta manera, el conocimiento de los distintos recursos y de su funcionalidad permitirá a los docentes hacer una mejor selección con miras a cumplir con los objetivos de aprendizaje. Un buen manejo del ecosistema virtual contribuirá a mejorar la percepción de los estudiantes que conciben la educación virtual como inferior a la educación presencial, lo cual ha obligado a algunos profesores a ofrecer una gran cantidad de material educativo para aliviar esa sensación. Además, ayudará a disminuir la sensación de sobrecarga académica percibida en comparación con ciclos presenciales (Lovón y Cisneros, 2020; Vicentini, 2020). Por otra parte, la creatividad y la innovación en este contexto ayudan a mantener los niveles atencionales y la motivación de los estudiantes, lo cual implica un desafío adicional en un ambiente en el que tanto la laptop como el dispositivo móvil son una fuente de reforzamiento inmediata de interacciones sociales, diversión, noticias, entre otros, al mismo tiempo que permiten el acceso a los recursos de aprendizaje y a las clases.

Por último, el haber mudado la educación al hogar de los estudiantes ha dificultado la interacción entre estudiantes, y entre ellos y los docentes (Canaza-Choque, 2020). Ello supone un reto para la educación, ya que, 
desde una perspectiva socioconstructivista, para que haya aprendizaje es necesaria la interacción con los otros para complejizar los conocimientos sobre el mundo (Coloma y Tafur, 1999). En ese sentido, la educación virtual no podría lograr sus objetivos si se da aisladamente, por lo cual debe establecerse una alianza pedagógica entre estudiantes y el docente encargado (Hassinger, 2020). Por consiguiente, la modalidad virtual necesita humanizarse aún más en un contexto de incertidumbre política, económica, social y sanitaria. En ese sentido, se podría hacer uso de la gran gama de herramientas que permiten comunicarnos durante la virtualidad, como las videollamadas, los foros, los chats grupales en las plataformas de aprendizaje y el correo institucional.

Aparte de los desafíos mencionados en este apartado, es necesario señalar algunos peligros que ha traído el cambio a la virtualidad. A raíz de la declaratoria del estado de emergencia, varias universidades tuvieron que adaptarse forzosamente a la modalidad virtual, aun cuando muchas de ellas no contaban con esa modalidad en el pasado (Lovón y Cisneros, 2020). Ello hizo que los servicios ofrecidos no fueran diseñados para la virtualidad, sino que representan lo que ha pasado a denominarse "educación remota por emergencia» (Soto y Valdivieso, 2020). Así, en algunos casos, se ha creído que el uso masivo de sesiones de videollamadas reemplazaría las clases presenciales, con la esperanza de que la virtualización no sea un medio oficial sino de tránsito hacia la educación presencial (Rodriguez-Segura et al., 2020). Esto se ha traducido en una enseñanza sin una adecuada organización, diseño y distribución de los recursos de aprendizaje (Álvarez, 2020), lo que es un riesgo para el aprendizaje de los estudiantes. Si dicha realidad se extiende a la mayoría de las universidades peruanas, podría resultar dañina a toda una generación de futuros profesionales y hacer que este tiempo de la universidad en la virtualidad sea cuestionable.

\section{Conclusiones}

En el presente ensayo se han presentado y discutido los factores importantes que influyen en la creación de experiencias educativas virtuales, y se han analizado los criterios que deben considerarse al ser implementadas, especialmente durante la etapa de pandemia. También se han establecido algunos desafíos que los docentes deben enfrentar para diseñar e implementar cursos virtuales en la universidad. A partir de toda la información presentada, se procede a señalar algunas conclusiones.

Primero, la innovación educativa involucra un cambio sustancial que rompe con la tradición de un espacio determinado. No necesariamente significa implementar algo nunca visto, puesto que la innovación puede 
usar prácticas educativas existentes, pero añade elementos nuevos, como las tecnologías de la información y la comunicación (González y Cruzat, 2019; Serdyukov, 2017).

Una segunda conclusión es que el docente puede utilizar los recursos disponibles para potenciar el aprendizaje de su alumnado a través de las diferentes herramientas virtuales existentes. No obstante, esto implica que los docentes ahora requieren de una continua actualización de sus conocimientos en el uso de metodologías aplicables a la virtualidad para el proceso de enseñanza, la planificación de contenidos digitales y el uso de las nuevas tecnologías (Freire et al., 2018). Adicionalmente, hay que tener en cuenta que las prácticas educativas de los docentes se basan en sus propias experiencias (Canedo et al., 2018); sin embargo, respecto a la modalidad virtual, no se encuentra una experiencia previa que les sirva como precedente. En ese sentido, muchos docentes no están familiarizados con las herramientas disponibles para el diseño de una clase. Así, las prácticas educativas de los docentes se basan en experiencias presenciales, y diseñar experiencias virtuales a partir de ellas podría generar un desfase en la experiencia educativa que se ofrece al estudiante.

Por otro lado, no todos los contenidos de un programa educativo pueden ser ofrecidos a través de una modalidad virtual. Si bien la educación virtual permite mayor autonomía a los estudiantes en su propio aprendizaje, así como flexibilidad para repasar los contenidos y hacer las actividades, la enseñanza de trabajos prácticos y clínicos sigue representando un reto que la educación virtual no puede cubrir (Ferri et al., 2020). Específicamente, disciplinas como los deportes, las ingenierías y los estudios médicos encuentran limitado su abordaje educativo por medio de la virtualidad tradicional (Heng y Sol, 2020). Por ejemplo, en las ciencias médicas se ha perdido la conexión con el ámbito clínico, ya que hay una imposibilidad de interactuar con pacientes reales, interacción que aporta de forma significativa en la formación del estudiante de ciencias de la salud (Núñez-Cortés, 2020).

Finalmente, a pesar del potencial de los entornos virtuales para la innovación y la enseñanza, se advierte una realidad contraproducente para el aprendizaje: que los docentes opten por trasladar las metodologías de enseñanza utilizadas en sus clases presenciales a un contexto virtual, sin adecuarla a las necesidades de la virtualidad en sí (Alves et al., 2017). Si esta realidad se hace presente en la educación universitaria, se puede generar un espacio de aprendizaje deficiente e incompleto.

Hay mucho que se puede hacer por la educación universitaria en esta pandemia y los docentes son piezas fundamentales para lograrlo. 
Sin embargo, es necesario ser conscientes de los desafíos que deben enfrentar para brindar una educación de calidad y saber cómo resolverlos adecuadamente.

\section{Contribución de autoría}

Ricardo Navarro es el autor principal del artículo y responsable de la estructura y redacción del documento. Renato López y Gloria Caycho participaron en la delimitación del objetivo y la redacción del artículo.

\section{Fuente de financiamiento}

Autofinanciado.

\section{Potenciales conflictos de interés}

Ninguno. 


\section{REFERENCIAS BIBLIOGRÁFICAS}

Álvarez, S. (2020). El impacto de la pandemia COVID-19 en la educación superior en el Perú y perspectivas de futuro. En Universidad de San Martín de Porres (Ed.), Educación y pandemia. Una visión desde la universidad (pp. 25-33). Cátedra Unesco Patrimonio Cultural y Turismo Sostenible.

Alves, P., Miranda, L. y Morais, C. (2017). The influence of virtual learning environments in students' performance. Universal Journal of Educational Research, 5(3), 517-527.

Arias, P. y Merino, M. (2016). Integración de las nuevas tecnologías al contexto educativo: Una visión desde el diseño curricular. Didasc@ lia:Didáctica y Educación, 7(6), 143-152.

Arkorful, V. y Abaidoo, N. (2015). The role of e-learning, advantages and disadvantages of its adoption in higher education. International Journal of Instructional Technology and Distance Learning, 12(1), 29-42.

Barari, N., RezaeiZadeh, M., Khorasani, A. y Alami, F. (2020). Designing and validating educational standards for E-teaching in virtual learning environments (VLEs), based on revised Bloom's taxonomy. Interactive Learning Environments, 1-13.

Bracho, K. y Bracho, M. (2020). COVID-19: Frente al desafío pedagógico de lo presencial a lo virtual. Hamut'ay, 7(2), 9-17.

Buabeng-Andoh, C., Yaokumah, W. y Tarhini, A. (2019). Investigating students' intentions to use ICT: A comparison of theoretical models. Education and Information Technologies, 24(1), 643-660.

Cable.co.uk. (2020). Worldwide broadband speed league 2020.

Canaza-Choque, F. (2020). Educación superior en la cuarentena global: disrupciones y transiciones. Revista Digital de Investigación en Docencia Universitaria, 14(2). https://doi.org/ 10.19083/ridu.2020.1315

Canedo, G., Guzmán, A., Cervantes, O. y Martínez, S. (2018). Prácticas educativas innovadoras. Experiencias para documentar y compartir. Instituto Nacional para la Evaluación de la Educación.

Cepal-Unesco. (2020, agosto). Informe COVID-19. La educación en tiempos de pandemia de COVID-19. Cepal-Unesco.

Chávez, J. y Jaramillo, C. (2014). El estudio de las prácticas educativas y su relevancia para el análisis de procesos de formación en docencia universitaria. Calidad en la educación, 41, 161-176.

Chen, L. (2016). A model for effective online instructional design. Literacy Information and Computer Education Journal, 6(2), 2303-2308. 
Coll, C., Onrubia, J. y Mauri, T. (2008). Ayudar a aprender en contextos educativos: el ejercicio de la influencia educativa y el análisis de la enseñanza [Supporting learning in educational contexts: The exercise of educational influence and the analysis of teaching]. Revista de Educación, 346, 33-70.

Coloma, C. y Tafur, R. (1999). El constructivismo y sus implicaciones en educación. Educación, 8(16), 217-244.

Deci, E. L. y Ryan, R. M. (2000). The «what» and «why» of goal pursuits: Human needs and the self-determination of behavior. Psychological Inquiry, $11(4), 227-268$.

Eom, S. (2019). The effects of student motivation and self-regulated learning strategies on student's perceived E-learning outcomes and satisfaction. Journal of Higher Education Theory \& Practice, 19(7).

Ferri, F., Grifoni, P. y Guzzo, T. (2020). Online learning and emergency remote teaching: Opportunities and challenges in emergency situations. Societies, 10(4). https://doi.org/10.3390/soc10040086

Figallo, F., Gonzáles, M. y Diestra, V. (2020). Perú: Educación superior en el contexto de la pandemia por el COVID-19. ESAL, Revista de Educación Superior en América Latina, 20-28.

Fırat, M., Kılınç, H. y Yüzer, T. V. (2018). Level of intrinsic motivation of distance education students in e-learning environments. Journal of Computer Assisted Learning, 34(1), 63-70.

Freire, J., Páez, M., Núñez, M., Narváez, M. y Infante, R. (2018). Curricular design a tool for educational achievement. Revista de Comunicación de la SEECl, (45), 75-86.

García, F., Corell, A., Abella-García, V. y Grande, M. (2020). Online assessment in higher education in the time of COVID-19. Education in the Knowledge Society, 21.

Gillies, R. M. (2003). Structuring cooperative group work in classrooms. International Journal of Educational Research, 39(1-2), 35-49.

González, C. y Cruzat, M. (2019). Innovación educativa: La experiencia de las carreras pedagógicas en la Universidad de Los Lagos, Chile. Educación, 28(55), 103-112. https://doi.org/10.18800/educacion.201902.005

Hassinger, Z. (2020). La educación virtual donde el estudiante es el protagonista. Una visión desde la universidad. En Universidad de San Martín de Porres (Ed.), Educación y pandemia. Una visión desde la universidad (pp. 45-53). Cátedra Unesco Patrimonio Cultural y Turismo Sostenible.

Heng, K. y Sol, K. (2020). Online learning during COVID-19: Key challenges and suggestions to enhance effectiveness. Cambodian Education Forum. 
Kumi-Yeboah, A. (2018). Designing a cross-cultural collaborative online learning framework for online instructors. Online Learning, 22(4), 181-201.

Lovón, M. y Cisneros, S. (2020). Repercusiones de las clases virtuales en los estudiantes universitarios en el contexto de la cuarentena por COVID-19: El caso de la PUCP. Propósitos y Representaciones, 8(3), 1-15.

Marquina, P., Avolio, B., Del Carpio, L., Fajardo, V., Chumbipuma, D. y Salas, A. (2020). Resultados del Ranking de Competitividad Mundial 2020. CENTRUM PUCP, Escuela de Negocios de la Pontificia Universidad Católica del Perú.

Martínez, C. (2008). La educación a distancia: sus características y necesidad en la educación actual. Educación, 17(33), 7-27.

Niño, S., Castellanos-Ramírez,J.y Patrón, F.(2021).Contraste de experiencias educativas de estudiantes universitarios en dos escenarios educativos: enseñanza en línea vs. enseñanza remota de emergencia. Revista de Educación a Distancia, 65(21). https://doi.org/10.6018/red.440731

Núñez-Cortés, J. (2020). Influencia de la pandemia por COVID-19 en la formación médica. Revista de Medicina y Cine, 16, 25-31. https://doi. org/10.14201/rmc202016e2531

Paechter, M. y Maier, B. (2010). Online or face-to-face? Students' experiences and preferences in e-learning. The Internet and Higher Education, 13(4), 292-297.

Parra, D., Zuñiga, C. y Lucio, E. (2020). Experiencias internacionales pedagógicas virtuales en la educación superior durante el confinamiento sanitario. Journal of Alternative Perspectives in the Social Sciences, 10(4), 971986.

Perales, M., Pedraza, L. y Moreno, P. (2019). Work-in-progress: Improving online higher education with virtual and remote labs [Conferencia]. IEEE Global Engineering Education Conference (EDUCON). Dubái. https://doi. org/10.1109/EDUCON.2019.8725272

Rahm, A. K., Töllner, M., Hubert, M. O., Klein, K., Wehling, C., Sauer, T., ... y Schultz, J. H. (2021). Effects of realistic e-learning cases on students' learning motivation during COVID-19. Plos One, 16(4), e0249425.

Ratnaningsih, S., Hady, Y., Dewi, R. S. y Zuhdi, M. (2020, octubre). The effectiveness of using Edmodo-Based E-learning in the blended learning process to increase student motivation and learning outcomes. En 2020 8th International Conference on Cyber and IT Service Management (CITSM) (pp. 1-5). IEEE.

Retnowati, E., Ayres, P. y Sweller, J. (2017). Can collaborative learning improve the effectiveness of worked examples in learning mathematics? Journal of Educational Psychology, 109(5), 666. 
Rodrigues, H., Almeida, F., Figueiredo, V. y Lopes, S. L. (2019). Tracking elearning through published papers: A systematic review. Computers \& Education, 136, 87-98.

Rodriguez-Segura, I., Zamora-Antuñano, M., Rodriguez-Resendiz, J., Paredes-García, W., Altamirano, J. y Cruz-Pérez, M. (2020). Teaching challenges in COVID-19 Scenary: Teams platform-based student satisfaction approach. Sustainability, 12(18). https://doi.org/10.3390/su12187514

Rosenberg, M. J. (2001). E-learning: estrategias para trasmitir conocimiento en la era digital. McGraw-Hill.

Sadik, A. (2017). Students' acceptance of file sharing systems as a tool for sharing course materials: The case of Google Drive. Education and Information Technologies, 22(5), 2455-2470.

Schreiber, L. M. y Valle, B. E. (2013). Social constructivist teaching strategies in the small group classroom. Small Group Research, 44(4), 395-411.

Serdyukov, P. (2017). Innovation in education: what works, what doesn't, and what to do about it? Journal of Research in Innovative Teaching \& Learning, 10(1), 4-33.

Soto, S. y Valdivieso, A. (2020). La gestión de la experiencia de la educación remota por emergencia y el nivel de satisfacción en los estudiantes de pregrado. [Tesis de pregrado, Pontificia Universidad Católica del Perú]. https:// tesis.pucp.edu.pe/repositorio/bitstream/handle/20.500.12404/18647/ Soto\%20Bermudez_Valdivieso\%20Chudan_Talledo\%20Peña_Gestión_ experiencia_educación\%20remota1.pdf?sequence=1\&isAllowed=y

Tarazona, J. (2012). Generalidades del diseño instruccional. Inventum, 12, 37-41.

Torres, T. y García, A. (2019). Reflexiones sobre los materiales didácticos virtuales adaptativos. Revista Cubana de Educación Superior, 38(3).

Tran, B. (2016). Educational experiences with traditional, online and hybrid learning environments. Journal of Education \& Social Policy, 3(3), 60-63.

Varas, H., Suárez, W., López, C. y Valdés, M. (2020). Educación virtual: factores que influyen en su expansión en América Latina. Utopía y Praxis Latinoamericana, 25, 21-40.

Vargas, J., Chiroque, E. y Vega, M. (2016). Innovación en la docencia universitaria. Una propuesta de trabajo interdisciplinario y colaborativo en educación superior. Educación, 25(48), 67-84. http://dx.doi.org/10.18800/ educacion.201601.004

Vialart, M. (2020). Estrategias didácticas para la virtualización del proceso enseñanza aprendizaje en tiempos de COVID-19. Educación Médica Superior, 34(3), 1-10. 
Vicentini, I. (2020). La educación superior en tiempos de COVID-19. Aportes de la Segunda Reunión del Diálogo Virtual con Rectores de Universidades Líderes de América Latina. Banco Interamericano del Desarrollo.

Xu, F. y Du, J. T. (2019). Examining differences and similarities between graduate and undergraduate students' user satisfaction with digital libraries. The Journal of Academic Librarianship, 45(6).

Recepción: 30/4/2021

Aceptación: 13/7/2021 